\title{
Nonlinear acoustic response of glasses in the tunneling model
}

\author{
Jürgen T. Stockburger* \\ II. Institut für Theoretische Physik, Universität Stuttgart, D-70550 Stuttgart, Germany \\ Milena Grifoni \\ Dipartimento di Fisica, Consorzio Interuniversitario Nazionale per la Fisica della Materia, \\ Università di Genova, I-16146 Genova, Italy \\ Maura Sassetti \\ Istituto di Fisica Ingegneria, Consorzio Interuniversitario Nazionale per la Fisica della Materia, \\ Università di Genova, I-16146 Genova, Italy \\ (Received 6 June 1994; revised manuscript received 11 October 1994)
}

\begin{abstract}
The nonlinear acoustic response of low-energy excitations in insulating glasses is investigated using the tunneling model. An explicit evaluation of the sound velocity shift is performed which covers the whole temperature range of recent vibrating-reed experiments. Our results are in excellent agreement with experimental findings. The comparison with recent findings on the nonlinear response in metallic glasses shows that the dynamics of the nonlinear driven tunneling system is qualitatively different for fermionic or bosonic environments. These differences are strongly reflected in the acoustic properties.
\end{abstract}

\section{INTRODUCTION}

Most of the low-temperature thermal and acoustic properties of amorphous solids have been successfully explained in terms of the phenomenological tunneling model (TM). ${ }^{1,2}$ The TM postulates the existence of lowenergy excitations of the disordered lattice that can be characterized by a double-well potential. At low temperatures these systems can be treated in the two-level approximation and are fully characterized by the bias energy $\hbar \epsilon_{0}$ and by the tunneling matrix element $\hbar \Delta$. Owing to the disordered structure, a broad distribution of these two parameters is assumed. Heuristic estimates suggest that this probability distribution is approximately independent of $\epsilon_{0}$ and inversely proportional to $\Delta$ over a wide parameter range.

The tunneling systems (TS's) determine the dispersion and absorption of sound in a way that is, in principle, similar to the way electronic excitations of an optical medium influence the propagation of light. The dynamics of tunneling systems, however, are strongly influenced by the relaxation mechanisms, which are given by their interaction with thermal phonons or, in the case of metallic glasses, conduction electrons. Our focus here will be mainly on the sound velocity; it is for this property rather than the acoustic absorption ${ }^{3,4}$ that a complete theoretical understanding seemed to be lacking.

A tacit assumption often made, and sometimes considered part of the "standard" TM, is the linear-response approximation for the interaction between the tunneling systems and the acoustic mode probed in the experiment. Recent vibrating-reed experiments, ${ }^{5,6}$ however, show a strong amplitude dependence of the acoustic response and even qualitative deviations from the TM predictions. The temperature at which the sound velocity reaches a maximum clearly depcnds on the applied strain field, and the temperature dependence below the maximum deviates from the expected logarithmic law. At very low temperatures the sound velocity is nearly constant. The question arises if these "anomalies" can still be explained by the tunneling model if the linearresponse approximation is abandoned.

In this paper we shall concentrate on insulating glasses, showing that the TM can quantitatively explain experimental data like the saturation at the lowest temperatures and the strain dependence of the maximum in the sound velocity.

It has been argued that the effects of the TS-phonon interaction can be treated perturbatively ${ }^{7}$ whenever the two-level approximation is valid. Thus, at the low frequency of interest in vibrating-reed studies, a natural approach is to treat the time dependence induced by the external field adiabatically ${ }^{3,4,8}$ and the frictional effects of the environment to lowest-order perturbation theory.

The case of phononic dissipation is to be contrasted with electronic dissipation in amorphous metals. In the latter case, the perturbative approach to the TS-electron interaction is known to fail for TS with very small energies because the fluctuations of the environment completely destroy coherence in the tunneling process. ${ }^{7}$

For the driven two-level system, this case has been treated in Ref. 9, using a controlled expansion for low frequencies. The results were applied to explain the discrepancies between previous theories and experiments probing the sound velocity of metallic glasses. ${ }^{10}$ The main findings of Refs. 9 and 10 will be mentioned in summary fashion in order to point out how the different dissipation mechanisms lead to qualitatively different TS dynamics and, ultimately, acoustic properties.

In Sec. II of this paper the nonlinear dynamics of the driven tunneling system interacting with phonons is discussed. A formal characterization of the reed vibration is outlined, and the relevant quantities of the TS non-

(c) 1995 The American Physical Society 
linear response are introduced. Following recent work by $\operatorname{Parshin}^{8}$ an explicit formal solution is derived in the adiabatic limit within the golden rule approach. We discuss the major differences compared to the results for ohmic dissipation, which is relevant when the dominant relaxation mechanism is given by inelastic scattering of conduction electrons.

In Sec. III we obtain explicit analytic expressions for the sound velocity shift that cover important parts of the relevant temperature range of vibrating-reed experiments. ${ }^{5}$ An inexpensive numerical evaluation applicable in the case of slow relaxation is also used, with details given in the Appendix. Finally we compare our results with experimental data in Sec. IV and present our main conclusions.

\section{ACOUSTIC NONLINEARITIES IN THE TUNNELING MODEL}

\section{A. Interaction of tunneling systems with sound waves}

The acoustic properties of glasses at temperatures below a few $\mathrm{K}$ are determined by the interaction of an extended acoustic mode with localized tunneling modes. Our subsequent discussion of this interaction, and the changes of the sound velocity it implies will be kept brief. A more detailed treatment, needed for a fully quantitative analysis of vibrating-reed experiments, is given in Ref. 10.

The dynamics of any tunneling mode is given by an Hamiltonian of the form

$$
H_{S}=H_{\mathrm{TS}}+H_{B}+H_{I}
$$

consisting of three terms for the tunneling system, environmental modes, and their mutual coupling. The tunneling Hamiltonian in its two-state approximation is characterized by the tunneling matrix element $\hbar \Delta$ and $\mathrm{a}$ time-dependent asymmetry $\epsilon(t)$,

$$
H_{\mathrm{TS}}=-\frac{\hbar}{2}\left[\Delta \sigma_{x}+\epsilon(t) \sigma_{z}\right]
$$

where $\sigma_{z}$ and $\sigma_{x}$ are Pauli's spin matrices. The asymmetry of a TS located at position $\mathbf{R}$,

$$
\epsilon(t)=\epsilon_{0}+\frac{\gamma}{2 \hbar} e(\mathbf{R}, t)=\epsilon_{0}+\hat{\epsilon} \cos \omega t
$$

acquires its time dependence from the strain field ${ }^{11} e(\mathbf{r}, t)$ of the macroscopically excited extended mode. A typical value of the coupling constant $\gamma$ is one or a few $\mathrm{eV}$.

In insulating glasses at low temperatures the relevant environmental modes are Debye phonons, ${ }^{12}$ giving $H_{B}$ the standard form

$$
H_{B}=\sum_{\mathbf{k}} \hbar \omega_{k} a_{\mathbf{k}}^{\dagger} a_{\mathbf{k}}
$$

where $a_{\mathbf{k}}^{\dagger}, a_{\mathbf{k}}$ are the phonon creation and annihilation operators, and where the wave vector $\mathbf{k}$ and the energy $\hbar \omega_{k}$ satisfy a linear dispersion relation. Finally, the coupling Hamiltonian

$$
H_{I}=\gamma \sigma_{z} X=\gamma \sigma_{z} \sum_{\mathbf{k}}\left(\frac{\hbar \omega_{k}}{2 V \rho v^{2}}\right)^{\frac{1}{2}}\left(a_{\mathbf{k}}^{\dagger} e^{-i \mathbf{k} \cdot \mathbf{R}}+a_{\mathbf{k}} e^{i \mathbf{k} \cdot \mathbf{R}}\right)
$$

describes a local interaction between the tunneling system and Debye phonons. Here $v$ is the sound velocity, $\gamma$ the coupling constant, and $\rho$ and $V$ are, respectively, the density and volume of the sample.

Apart from the explicit time dependence of the asymmetry frequency $\epsilon(t)$, the Hamiltonian (2.1) with (2.4) and (2.5) is the well-known spin-boson Hamiltonian, ${ }^{7,13}$ which describes a general two-state system coupled bilinearly to an environment of bosonic modes with a quasicontinuous spectrum.

The dynamical variable of interest for the tunneling system is its normalized position operator $\sigma_{z}$, which determines the amplitude and phase of the force acting back on the acoustic mode through the interaction potential

$$
U_{I}(e(\mathbf{r}))=-\frac{\gamma}{\Delta V} \sum_{j} e\left(\mathbf{R}_{j}\right)\left\langle\sigma_{z}^{(j)}\right\rangle_{t}
$$

where the summation is carried out over the tunneling systems contained in the volume element $\Delta V$, and $\left\langle\sigma_{z}^{(j)}\right\rangle_{t}$ denotes a quantum statistical expectation value.

The case that is relevant here is monochromatic driving (continuous-wave conditions), which allows one to expand $\left\langle\sigma_{z}^{(j)}\right\rangle_{t}$ in a Fourier series. Rewriting the fundamental frequency component in terms of the generalized nonlinear susceptibility ${ }^{10}$

$$
\tilde{\chi}(\omega ; \hat{\epsilon})=\frac{\omega}{2 \pi \hbar \hat{\epsilon}} \int_{0}^{2 \pi / \omega} d t \exp (i \omega t)\left\langle\sigma_{z}\right\rangle_{t},
$$

one obtains the decomposition

$$
\left\langle\sigma_{z}^{(j)}\right\rangle_{t}=\hbar \hat{\epsilon} \tilde{\chi}(\omega ; \hat{\epsilon}) e^{-i \omega t}+\text { c.c. }+ \text { higher harmonics }
$$

In the context of a vibrating-reed experiment, the higherfrequency components can be discarded because the resonances of bending vibrations do not occur at integral multiples of the fundamental frequency. This makes it easy to see how the presence of the TS modifies the elastic properties of the sample. The stress component $s(\mathbf{r}, t)$ induced by the tunneling systems is given by the derivative of $U_{I}(e)$. With

$$
e(\mathbf{r}, t)=\hat{e}(\mathbf{r}) \cos \omega t
$$

it can be cast in the form

$$
s(\mathbf{r}, t)=\kappa(\omega) \hat{e}(\mathbf{r}) \exp (-i \omega t) / 2+\text { c.c. },
$$

where the complex-valued elastic constant $\kappa(\omega)$ is related to the nonlinear susceptibility by

$$
\kappa(\omega)=4 \gamma^{2} \bar{P} \bar{\chi}(\omega)
$$




$$
\bar{\chi}(\omega)=\hbar \int_{\Delta_{\min }}^{\Delta_{\max }} \frac{d \Delta}{\Delta} \int_{0}^{\epsilon_{\max }} d \epsilon_{0} \tilde{\chi}\left(\Delta, \epsilon_{0} ; \hat{\epsilon}, \omega\right) .
$$

These integrations represent the summation over tunneling systems when the standard probability distribution of the TS parameters $\Delta$ and $\epsilon_{0}$, and their positions

$$
P\left(\epsilon_{0}, \Delta\right) d \Delta d \epsilon_{0} d^{3} r=\bar{P} \Delta^{-1} d \Delta d \epsilon_{0} d^{3} r
$$

is used.

It is obvious that the contribution $\kappa(\omega)$ to the elastic constant introduces a change of the sound velocity and a damping of the extended mode. The relative shift of the sound velocity and the dimensionless damping constant $Q^{-1}$ are given by

$$
\begin{aligned}
\frac{\Delta v}{v} & =-2 C \operatorname{Re} \bar{\chi}(\omega), \\
Q^{-1} & =4 C \operatorname{Im} \bar{\chi}(\omega)
\end{aligned}
$$

with $C=\gamma^{2} \bar{P} / \rho v^{2}$.

\section{B. TS dynamics in the adiabatic limit}

To evaluate the expectation value $\left\langle\sigma_{z}\right\rangle$ for low driving frequencies, it is convenient to perform the unitary transformation

$$
\begin{gathered}
R(t)=\exp \left\{(i / 2) \theta(t) \sigma_{y}\right\} \\
\tan \theta(t)=\frac{\Delta}{\epsilon(t)}
\end{gathered}
$$

which diagonalizes the system Hamiltonian $H_{\mathrm{TS}}(t)$ given in (2.2) at any times $t$. The resulting transformed Hamiltonian is

$$
\begin{aligned}
\tilde{H} & =R H_{S} R^{-1} \\
& =-\frac{E}{2} \sigma_{z}+\gamma X\left(\cos \theta \sigma_{z}-\sin \theta \sigma_{x}\right)+H_{B},
\end{aligned}
$$

where $E(t)=\hbar \sqrt{\Delta^{2}+\epsilon(t)^{2}}$ is the time-dependent level splitting. The expectation value $\left\langle\sigma_{z}\right\rangle_{t}$ is related to the transformation by

$$
\begin{aligned}
\left\langle\sigma_{z}\right\rangle_{t} & =\operatorname{tr}\left\{\rho(t) \sigma_{z}\right\} \\
& =\cos \theta \operatorname{tr}\left\{\tilde{\rho}(t) \sigma_{z}\right\}-\sin \theta \operatorname{tr}\left\{\tilde{\rho}(t) \sigma_{x}\right\},
\end{aligned}
$$

where $\tilde{\rho}(t)=R \rho(t) R^{-1}$, and $\rho(t)$ is the density matrix of the system. The evolution of the transformed density matrix $\tilde{\rho}(t)$ is now governed by

$$
H_{\mathrm{eff}}=\tilde{H}(t)+\dot{R} R^{-1}
$$

where the additional term $\dot{R} R^{-1}$ is due to the explicit time dependence of the transformation.

The frequency of vibrating-reed experiments is so low that one would naturally assume that the adiabatic limit can be applied, ${ }^{3,4}$ i.e., the term $\dot{R} R^{-1}$ can be neglected.
Nevertheless, we would like to point out which constraints must be imposed on the system parameters in order to ensure that this limit is applicable. In addition to the obvious condition $\hbar \omega \ll E$, one has to make sure that the contribution $\dot{R} R^{-1}$ to the Hamiltonian (2.20) can be treated as a small perturbation to $\tilde{H}$. This is the case if the operator norm of $\dot{R} R^{-1}$ is small compared to the level spacing $E(t)$, i.e.,

$$
\hbar \dot{\theta} \equiv \frac{\hbar^{3} \Delta \dot{\epsilon}}{E^{2}} \ll E
$$

For realistic parameters of vibrating-reed experiments, one normally finds $\omega \ll \hat{\epsilon}$. In this case the condition $\hbar \omega \ll E$ is always a weaker constraint than (2.21).

In the following we shall assume $\Delta_{\min }^{2}>\omega \hat{\epsilon}$, where $\Delta_{\min }$ is the lower bound for $\Delta$ in the TS distribution function. The adiabatic limit is then valid for all tunneling systems.

\section{Time-dependent relaxation}

Let us now discuss the frictional influences of the thermal bath on the TS dynamics. It was argued earlier that the effect of the TS-phonon interaction on the dynamics can be treated as a perturbation whenever the two-state approximation of the tunneling system is applicable. ${ }^{7}$

Within the adiabatic limit, the golden-rule approach can be applied as in the case of static asymmetry. Equation (2.19) then assumes the form

$$
\begin{aligned}
\left\langle\sigma_{z}\right\rangle_{t} & =\operatorname{tr}\left\{\rho(t) \sigma_{z}\right\} \\
& =\cos \theta \operatorname{tr}\left\{\tilde{\rho}(t) \sigma_{z}\right\}=\frac{\hbar \epsilon(t)}{E(t)} N(t),
\end{aligned}
$$

where $N(t)=n_{-}(t)-n_{+}(t)$ is the difference of the occupation numbers of the two levels, and its dynamics are governed by the rate equation

$$
\dot{N}(t)=-\Gamma(t)\left[N(t)-N_{\mathrm{eq}}(t)\right] .
$$

Second-order perturbation theory in $\gamma$ leads to the expression

$$
\Gamma(t)=\frac{\Delta^{2} E \gamma^{2}}{2 \pi \hbar^{2} \rho v^{5}} \operatorname{coth} \frac{E}{2 k T} \equiv \Delta^{2} A E \operatorname{coth} \frac{E}{2 k T}
$$

for the rate, ${ }^{12}$ and from the equilibrium occupation numbers of the TS one obtains $N_{\text {eq }}(t)=\tanh \frac{E(t)}{2 k T}$. A formal solution of (2.23) can easily be given in terms of quadratures; the explicit form of the continuous-wave solution is

$$
N(t)=\int_{-\infty}^{t} d t^{\prime} \exp \left(-\int_{t^{\prime}}^{t} d t^{\prime \prime} \Gamma\left(t^{\prime \prime}\right)\right) \Gamma\left(t^{\prime}\right) N_{\mathrm{eq}}\left(t^{\prime}\right) .
$$

It is interesting to compare the result given by (2.22) and (2.25) to that found in previous work, 9,10 where the dynamics of driven TS in metals and their effect on acous- 
tic properties of metallic glasses were studied. For any $\Delta$ smaller than the frequency scale $\alpha k T / \hbar$, where $\alpha$ is the dimensionless friction constant, the golden-rule approximation cannot be applied to the system-environment interaction. $^{14,7,13}$ A careful path-integral evaluation of $\left\langle\sigma_{z}\right\rangle_{t}$ using a controlled expansion for frequencies $\omega$ lower than $\alpha k T / \hbar$ yields

$$
\left\langle\sigma_{z}\right\rangle_{t}=\int_{-\infty}^{t} d t^{\prime} \exp \left(-\int_{t^{\prime}}^{t} d t^{\prime \prime} \Gamma_{m}\left(t^{\prime \prime}\right)\right) \Gamma_{m}\left(t^{\prime}\right) P_{\mathrm{eq}}\left(t^{\prime}\right)
$$

for $\hbar \Delta<k T$. This expression obeys a differential equation analogous to $(2.23)$, with the notable difference that the dynamical variable now is the coordinate of the tunneling system, not the occupation of energy levels. The rate assumes the form

$$
\Gamma_{m}(t)=\pi \alpha \Delta^{2} \frac{\epsilon(t)}{(2 \pi \alpha k T / \hbar)^{2}+\epsilon^{2}(t)} \operatorname{coth} \frac{\hbar \epsilon(t)}{2 k T},
$$

where the presence of $\alpha$ in the denominator reflects the nonperturbative nature of the approach. The normalized equilibrium position $P_{\text {eq }}$ is given by

$$
P_{\text {eq }}(t)=\tanh \frac{\hbar \epsilon(t)}{2 k T} .
$$

In spite of these formal analogies, there are notable differences between the two cases. The level splitting $E(t)$ that determines the equilibrium expression $N_{\text {eq }}$ in the goldenrule case is always positive. A consequence of this is the fact that almost all TS's are frozen in the ground state $\left(N_{\text {eq }}=1\right)$ for $\hbar \hat{\epsilon} \gg k T$. The equilibrium position $P_{\text {eq }}$ obtained by evaluating (2.28) in this limit, on the other hand, is a step function oscillating between +1 and -1 .

The behavior for small $\Delta$, which dominate the TS parameter distribution, is also markedly different. Small values of $\Delta$ imply a small relaxation rate $\Gamma \ll \omega$, giving a time-independent leading term in the formal expressions (2.25) and (2.26). In the case of amorphous metals, this means that the TS remains at rest and does not influence the acoustic properties. For insulating glasses, however, the time-dependent prefactor $\hbar \epsilon(t) / E(t)$ in (2.22) leads to a nonvanishing response even when the occupation numbers are constant. These differences between electronic and phononic environments are reflected in strikingly different result for the sound velocity, as we are about to point out in Sec. III.

\section{PARAMETER AVERAGING OF THE TS RESPONSE}

\section{A. Linear response}

Theoretical results for the temperature dependence of the sound velocity and damping have been derived from the tunneling model in the linear-response limit for insulating glasses earlier by Jäckle, ${ }^{12}$ and Hunklinger and Raychaudhury ${ }^{15}$ and later also for amorphous metals by
Golding, Graebner, and Kane, ${ }^{16}$ and Black. ${ }^{17}$ In both cases two distinct temperature ranges with different underlying dynamics of the tunneling systems are found. In the limit of low temperatures, the relaxation of tunneling systems towards thermal equilibrium slows down, which leads to an absorption that vanishes for $T \rightarrow 0$,

$$
Q^{-1}=\frac{\pi^{4}}{6} C A \frac{k^{3} T^{3}}{\hbar^{2} \omega} .
$$

The sound velocity varies logarithmically with temperature,

$$
\frac{\Delta v}{v}=C\left(\ln \frac{k T}{E_{\max }}-\gamma_{E}+\ln \frac{\pi}{2}\right),
$$

where $\gamma_{E} \approx 0.577$ is Euler's constant, and $E_{\max }$ is the high-energy cutoff of the level splitting. For high temperatures, on the other hand, the wide distribution of TS parameters leads to a distribution of relaxation times extending over a huge range. In this region, one finds a universal constant

$$
Q^{-1}=\frac{\pi}{2} C
$$

for the damping, and again a logarithmic law

$$
\frac{\Delta v}{v}=\frac{n}{2} C \ln k T+\text { const }
$$

for the sound velocity. The values $n=-1$ for insulating glasses and $n=+1$ for amorphous metals reflect the different temperature dependence of the rates for relaxation mediated by thermal phonons or conduction electrons.

For insulating glasses the two regimes are separated by the temperature scale

$$
T_{\omega}=\left(\frac{\hbar^{2} \omega}{A k^{3}}\right)^{\frac{1}{3}}
$$

which lies within the experimentally relevant temperature range even for the very low frequencies of vibratingreed measurements.

\section{B. Slow relaxation}

The formal solution (2.25) of the equation of motion (2.23) is significantly simplified when the rate $\Gamma(t)$ is much less than the frequency $\omega$ at all times $t$ for those tunneling systems that can be thermally excited. This condition is always fulfilled in the parameter range

$$
T \ll T_{\omega}, \quad \hbar \hat{\epsilon} T^{2} \ll k T_{\omega}^{3} .
$$

It is convenient for the following to sum over periodicity intervals in the integrand of (2.25), yielding the expression

$$
N(t)=\frac{\int_{t-2 \pi / \omega}^{t} d t^{\prime} \exp \left\{-\int_{t^{\prime}}^{t} d t^{\prime \prime} \Gamma\left(t^{\prime \prime}\right)\right\} \Gamma\left(t^{\prime}\right) N_{\mathrm{eq}}\left(t^{\prime}\right)}{1-\exp \left\{-\int_{0}^{2 \pi / \omega} d t^{\prime} \Gamma\left(t^{\prime}\right)\right\}}
$$


which is still exact. Taking only the leading terms of both numerator and denominator of (3.7) in the limit $\Gamma \ll \omega$, one gets the time-independent result

$$
N(t)=\frac{\int_{0}^{2 \pi / \omega} d t^{\prime} \Gamma\left(t^{\prime}\right) \tanh \left[E\left(t^{\prime}\right) / 2 k T\right]}{\int_{0}^{2 \pi / \omega} d t^{\prime} \Gamma\left(t^{\prime}\right)}=\mathrm{const}
$$

for the cw limit. Obviously, the time dependence of $\left\langle\sigma_{z}(t)\right\rangle$ as given by (2.22) now originates only from the prefactor $\hbar \epsilon(t) / E(t)$.

Except for the integration boundaries of the parameter distribution, there is no characteristic energy scale of the ensemble of tunneling systems. Among the possible parameters that could define a threshold for nonlinear effects, the thermal energy $k T$ remains as the only likely candidate. Deviations from the linear response limit can therefore be expected to appear in the acoustic properties when the condition $\hbar \hat{\epsilon} \ll k T$ is violated.

Taking the opposite limit $\hbar \hat{\epsilon} \gg k T$, one can simplify (3.8) and explicitly evaluate $\Delta v / v$. The population of the upper energy level is always very small for $\hbar \Delta>2 k T$, i.e., we can set $N(t) \approx 1$ in this case. Otherwise we have $\hbar \Delta<2 k T \ll \hat{\epsilon}$ and can approximate $E(t)=\hbar|\epsilon(t)|$. Thus, one is lead to divide $\bar{\chi}$ into two terms,

$$
\begin{aligned}
\bar{\chi} \approx & \bar{\chi}_{1}+\bar{\chi}_{2} \\
\bar{\chi}_{1}= & \frac{\omega}{2 \pi \hat{\epsilon}} \int_{2 k T / \hbar}^{\Delta_{\max }} \frac{d \Delta}{\Delta} \int_{0}^{\epsilon_{\max }} d \epsilon_{0} \int_{0}^{2 \pi / \omega} d t \\
& \times e^{i \omega t} \frac{\epsilon(t)}{\sqrt{\Delta^{2}+\epsilon^{2}(t)}} \\
\bar{\chi}_{2}= & \frac{\omega}{2 \pi \hat{\epsilon}} \int_{\Delta_{\min }}^{2 k T / \hbar} \frac{d \Delta}{\Delta} \int_{0}^{\epsilon_{\max }} d \epsilon_{0} \int_{0}^{2 \pi / \omega} d t e^{i \omega t} \operatorname{sgn} \epsilon(t) \\
& \times \frac{\int_{0}^{2 \pi / \omega} d t^{\prime} \Gamma_{0}\left(t^{\prime}\right) \tanh \left|\hbar \epsilon\left(t^{\prime}\right) / 2 k T\right|}{\int_{0}^{2 \pi / \omega} d t^{\prime} \Gamma_{0}\left(t^{\prime}\right)}
\end{aligned}
$$

where $\Gamma_{0}(t)=\epsilon(t) \operatorname{coth}[\hbar \epsilon(t) / 2 k T]$. Rewriting the first term as

$$
\bar{\chi}_{1}=\frac{2}{\pi} \int_{0}^{\pi / 2} d x \cos x \int_{2 k T / \hbar}^{\Delta_{\max }} \frac{d \Delta}{\Delta} \int_{\epsilon_{\max }-\hat{\epsilon} \cos x}^{\epsilon_{\max }+\hat{\epsilon} \cos x} \frac{d \epsilon_{0}}{\hat{\epsilon}} \frac{\epsilon_{0}}{\sqrt{\Delta^{2}+\epsilon_{0}^{2}}}
$$

then expanding for $\hat{\epsilon} \ll \epsilon_{\max }$ and using the common convention $\Delta^{2}+\epsilon_{0}^{2}<\hbar^{-2} E_{\max }^{2}$ for the high-frequency cutoff of the TS parameters leads to the analytic result

$$
\bar{\chi}_{1}=\frac{1}{2}\left(\ln \frac{E_{\max }}{k T}-1\right) \text {. }
$$

For the second term we find that only TS with asymmetries $\epsilon_{0}<\hat{\epsilon}$ contribute, since $\operatorname{sgn} \epsilon(t) \equiv 1$ when $\epsilon_{0}>\hat{\epsilon}$. This yields

$$
\operatorname{Re} \bar{\chi}_{2}=\left[\frac{1}{2}-\frac{16 a}{\pi}\left(\frac{k T}{\hbar \hat{\epsilon}}\right)^{2}\right] \ln \frac{2 k T}{\hbar \Delta_{\min }}
$$

where $a=\int_{0}^{1} d b\left(\int_{0}^{2 \pi} d x|b+\cos x|\right)^{-1}=0.216$. Adding the two contribution we finally find

$$
\frac{\Delta v}{v}=C\left[\ln \frac{\hbar \Delta_{\min }}{2 E_{\max }}+1+\frac{32 a}{\pi}\left(\frac{k T}{\hbar \hat{\epsilon}}\right)^{2} \ln \frac{2 k T}{\hbar \Delta_{\min }}\right]
$$

for the saturation regime.

A similar result that did not explicitly include the temperature-independent terms has been reported by Parshin. ${ }^{8}$ Although empirical data of the sound velocity can only be obtained as a shift with respect to an arbitrarily chosen reference value, these terms do have experimental significance. The reason for this is the obvious fact that a unique reference must be used consistently for both the linear-response and the saturation regime. Having chosen the commonly used form (3.2) for the linear-response result, one cannot eliminate the leading term of (3.15).

The next-to-leading term of (3.7) in the limit $\Gamma \ll \omega$ yields the dominant contribution to the damping constant $Q^{-1}$. An evaluation of the parameter averaging similar to the above calculation (see also Refs. 3 and 4) yields a damping constant that is reduced by a factor $k T / \hbar \hat{\epsilon}$ from its linear-response value $Q_{\mathrm{lr}}^{-1}$ as given in (3.1),

$$
Q^{-1} \approx \frac{k T}{\hbar \hat{\epsilon}} Q_{\mathrm{lr}}^{-1}
$$

Obviously, the linear and saturation results match at the saturation threshold $\hbar \hat{\epsilon}=k T$. For the sound velocity, on the other hand, one finds a gap of the size $C \ln \left(\hat{\epsilon} / \Delta_{\min }\right)$ between the two results when extrapolating both the leading term of (3.15) and the linear response result (3.2) into the region $\hbar \hat{\epsilon} \approx k T$ (Fig. 1). This is an indication of a fairly wide intermediate parameter range where the full expression (3.8) for the occupation numbers must be used for a correct prediction of the sound velocity.

\section{Crossover to fast relaxation}

At a temperature $T_{c o}$ in the vicinity of $T_{\omega}$, the sound velocity assumes its maximum value. In most experiments both the position and the height of the maximum depend on the strain amplitude, which clearly indicates that the linear response approximation is invalid. Moreover, there is a wide distribution of relaxation rates $\Gamma(t)$ at temperatures near or above $T_{\omega}$, which makes a theoretical analysis of this situation in general very difficult.

In many cases, however, the maximum is found in a parameter region not too far from the linear response limit, i.e, for $\hbar \hat{\epsilon} \ll 2 k T$ and $\hbar \hat{\epsilon} \ll 2 k T\left(T_{\omega} / T\right)^{3 / 2}$. An approxi- 


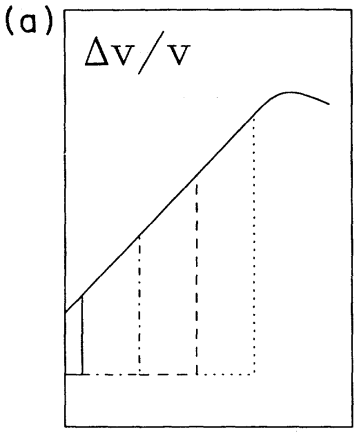

$\log \mathrm{T}$

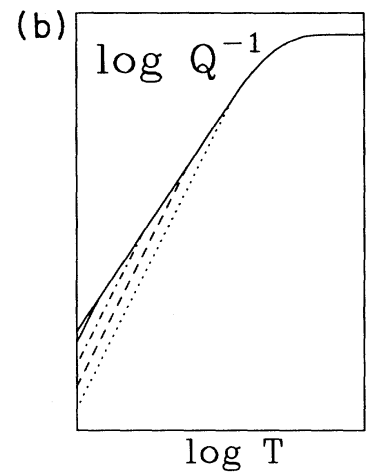

FIG. 1. (a) Discrepancy between results for the velocity shift $\Delta v / v$ in the linear-response and saturation limits at the nonlinearity threshold $k T \approx \hbar \hat{\epsilon}$. (b) Smooth transition between linear and saturation results for the damping constant $Q^{-1}$. In both frames the solid uppermost curve represents the linear response. Other curves correspond to the leading terms of (3.15) and (3.16) for increasing values of $\hbar \hat{\epsilon}$ (left to right).

mate expansion of the sound velocity for these limits will be presented in the following. It is convenient here to split both parameter integrations at $\hat{\epsilon}$, thus rewriting $\bar{\chi}$ as a sum of four terms

$$
\bar{\chi}=\bar{\chi}^{a}+\bar{\chi}^{b}+\bar{\chi}^{c}+\bar{\chi}^{d},
$$

where

$$
\begin{gathered}
\bar{\chi}^{a}=\int_{\hat{\epsilon}}^{\Delta_{\max }} \frac{d \Delta}{\Delta} \int_{\hat{\epsilon}}^{\epsilon_{\max }} d \epsilon_{0} \tilde{\chi}, \\
\bar{\chi}^{b}=\int_{\hat{\epsilon}}^{\Delta_{\max }} \frac{d \Delta}{\Delta} \int_{0}^{\hat{\epsilon}} d \epsilon_{0} \tilde{\chi}, \\
\bar{\chi}^{c}=\int_{\Delta_{\min }}^{\hat{\epsilon}} \frac{d \Delta}{\Delta} \int_{\hat{\epsilon}}^{\epsilon_{\max }} d \epsilon_{0} \tilde{\chi}, \\
\bar{\chi}^{d}=\int_{\Delta_{\min }}^{\hat{\epsilon}} \frac{d \Delta}{\Delta} \int_{0}^{\hat{\epsilon}} d \epsilon_{0} \tilde{\chi} .
\end{gathered}
$$

To evaluate (3.17) we need to consider which energy scale dominates the level splitting in each of the four parameter regions considered. For instance, in the integration range of the TS parameters that contribute to $\bar{\chi}^{a}, \hbar \hat{\epsilon}$ is the smallest energy scale. In this region one can apply the linear response approximation, i.e., replace $\tilde{\chi}$ by the linear susceptibility,

$$
\begin{aligned}
\tilde{\chi} \approx \chi \equiv & \frac{1}{2} \frac{\hbar^{2} \Delta^{2}}{E_{0}^{3}} \tanh \left(\frac{E_{0}}{2 k T}\right) \\
& +\frac{1}{4 k T} \frac{1}{\cosh ^{2}\left(E_{0} / 2 k T\right)} \frac{\hbar^{2} \epsilon_{0}^{2}}{E_{0}^{2}} \frac{1}{1+i \Gamma_{I} / \omega}
\end{aligned}
$$

with $E_{0}=\hbar \sqrt{\Delta^{2}+\epsilon_{0}^{2}}$ and $\Gamma_{I}=\Delta^{2} A E_{0} \operatorname{coth} \frac{E_{0}}{2 k T}$ being the linear intrinsic level splitting and the relaxation rate.
The first and second term of (3.22) are usually called the "resonant" and "relaxational" parts of the susceptibility and are treated separately in the following. Approximating tanh $x=\Theta(x-1)+x \Theta(1-x)$ and $\cosh ^{-2} x=$ $\Theta(1-x)$ for all corrections to the linear-response result (3.2), we get

$$
\begin{aligned}
\bar{\chi}_{\mathrm{res}}^{a}= & \frac{1}{2}\left\{\left(\ln \frac{E_{\max }}{k T}+\gamma_{E}-\ln \frac{\pi}{2}\right)+\frac{\hbar \hat{\epsilon}}{2 k T} \ln \frac{\hbar \hat{\epsilon}}{2 k T}\right\} \\
& +O(\hbar \hat{\epsilon} / 2 k T) .
\end{aligned}
$$

Using the same approximation, and introducing the dimensionless variable $\eta=\left(2 T / T_{\omega}\right)^{3}, \operatorname{Re} \bar{\chi}_{\text {rel }}^{a}$ assumes the form

$$
\operatorname{Re} \bar{\chi}_{\mathrm{rel}}^{a}=\int_{0}^{1} d x \int_{0}^{\sqrt{1-x^{2}}} \frac{d y}{2 y} \frac{y^{4} \eta^{2}}{1+y^{4} \eta^{2}} \frac{x^{2}}{x^{2}+y^{2}},
$$

where terms of order $(\hbar \hat{\epsilon} / 2 k T)^{2}$ or $\eta(\hbar \hat{\epsilon} / 2 k T)^{2}$ have been neglected. The integrations in (3.24) yield the approximate result

$$
\operatorname{Re} \bar{\chi}_{\text {rel }}^{a}=-\frac{1}{2}\left(I(\eta)-\frac{1}{4} \ln \left(1+\eta^{2}\right)\right),
$$

where the function $I$ is

$$
\begin{aligned}
I(x)= & \frac{1}{4 x}(\arctan x-x)+\frac{\pi}{2} \\
& -\frac{\pi}{4 \sqrt{2 x}}\left(\operatorname{arctanh} \frac{\sqrt{2 x}}{1+x}+\arctan \frac{\sqrt{2 x}}{1-x}\right) .
\end{aligned}
$$

Let us now calculate the other three terms along the same lines. In the evaluation of $\bar{\chi}^{b}, \hbar \Delta$ is the dominant energy scale. The parameter integrations are thus evaluated using $E(x) \approx \hbar \Delta$, which yields

$$
\operatorname{Re} \bar{\chi}^{b}=-\frac{1}{2} \frac{\hbar \hat{\epsilon}}{2 k T} \ln \frac{\hbar \hat{\epsilon}}{2 k T}+O\left(\frac{\hbar \hat{\epsilon}}{2 k T}\right) .
$$

In the parameter region $\Delta<\hat{\epsilon}<\epsilon_{0}$ relevant to $\bar{\chi}^{c}$, one finds $E(x) \approx \hbar \epsilon(x)$. This leads to

$$
\operatorname{Re} \bar{\chi}^{c}=O\left((\hbar \hat{\epsilon} / 2 k T)^{2}\right)+O\left(\eta(\hbar \hat{\epsilon} / 2 k T)^{2}\right) .
$$

Finally, the parameter region $\Delta<\hat{\epsilon}, \epsilon_{0}<\hat{\epsilon}$ that is to be considered for $\bar{\chi}^{d}$ shows pronounced nonlinear behavior. This term is dominated by TS with very small tunneling matrix elements. Here the asymmetry changes its sign periodically, and $\operatorname{Re} \tilde{\chi}$ remains finite even for $\Delta \rightarrow 0$. Observing that the parameter distribution $P\left(\Delta, \epsilon_{0}\right)$ diverges in this limit, we obtain a contribution to $\bar{\chi}^{d}$, which depends logarithmically on $\Delta_{\min }$,

$$
\operatorname{Re} \bar{\chi}^{d}=\frac{32}{9 \pi^{2}} \frac{\hbar \hat{\epsilon}}{2 k T} \ln \frac{\hat{\epsilon}}{\Delta_{\min }}+O\left(\frac{\hbar \hat{\epsilon}}{2 k T}\right) .
$$

Adding these terms, one gets the final result 


$$
\begin{aligned}
\frac{\Delta v}{v}= & C\left[\ln \frac{k T}{E_{\max }}-\gamma_{E}+\ln \frac{\pi}{2}\right. \\
& -\frac{1}{4} \ln \left(1+\eta^{2}\right)+I(\eta) \\
& \left.-\frac{\hat{\epsilon}}{2 k T} \frac{64}{9 \pi^{2}} \ln \frac{\hat{\epsilon}}{\Delta_{\min }}\right]
\end{aligned}
$$

where terms that vanish in the limit $\Delta_{\min } \ll \hat{\epsilon}$ and near the linear response limit have been neglected.

It is obvious from (3.30) that the lower bound $\Delta_{\min }$ in the TS parameter distribution plays an important role also in the crossover region, as we observed already in the saturation regime. The leading nonlinear correction is again dominated by $\mathrm{TS}$ with very small relaxation rates.

\section{RESULTS AND CONCLUSIONS}

The different parameter regimes, which we have discussed, yield a fairly complete picture that can be compared to experimental results. ${ }^{18}$ Figure 2 shows graphs of the temperature dependence of the sound velocity for $\Delta_{\min }=10^{-8} \mathrm{~K}$ and $T_{\omega}=30 \mathrm{mK}$ using both our analytical results (3.15) and (3.30) as well as the numerical evaluation of the sound velocity with $N(t)$ given by Eq. (3.8). The computational cost of evaluating the sound velocity repeatedly for varying values of $T, \hat{\epsilon}, \Delta_{\min }$, and $\Delta_{\max }$ can be kept at a minimum by using scaled variables and tabulating two auxiliary functions (see the Appendix). The curves show a close resemblance to the experimental results of Esquinazi, König, and Pobell. ${ }^{5}$ For comparison, the $\ln T$ law (3.4), which linear response theory predicts for $T \ll T_{\omega}$, is indicated by a dotted line.

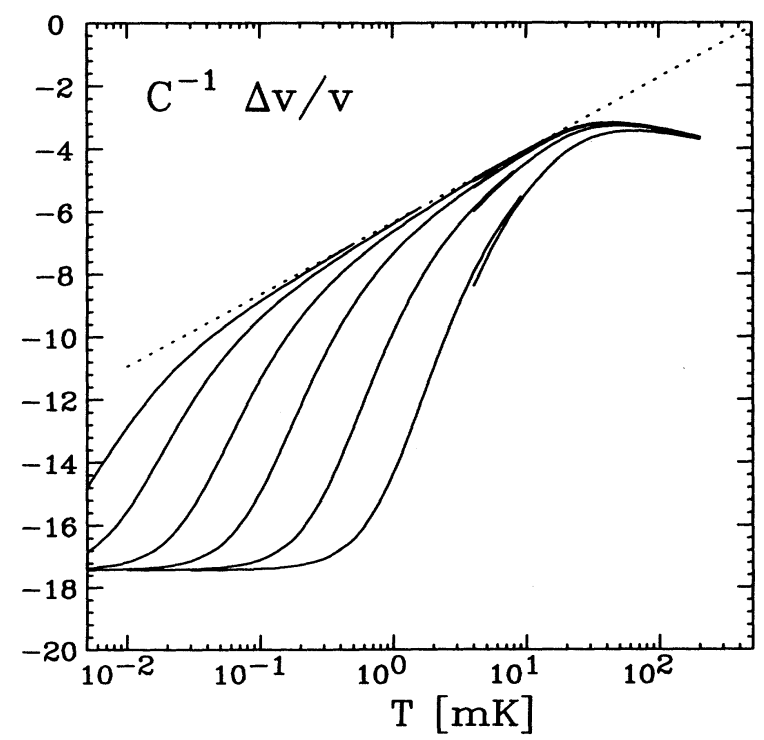

FIG. 2. Temperature dependence of the sound velocity of a insulating glass for different strain levels. Curves are for $\hbar \hat{\epsilon} / k=0.01,0.03,0.1,0.3,1$, and $3 \mathrm{mK}$ (left to right). Other parameters are $\Delta_{\min }=10^{-8} \mathrm{~K}$ and $T_{\omega}=30 \mathrm{mK}$.
Around $k T \approx \hbar \hat{\epsilon}$, the gap between these two asymptotic results leads to a very pronounced temperature dependence of the sound velocity, which is far stronger than it would be in the linear response approximation. The transition regime where none of the two limiting cases constitutes a good approximation is quite wide. This is confirmed by experimental results, ${ }^{5}$ which show a regime of visible nonlinear behavior that extends to higher temperatures in the sound velocity data than in the absorption data. For the damping, the transition from linear to nonlinear behavior is much smoother, as one would naturally conclude from a comparison of (3.1) and (3.16), or from the sketch in Fig. 1.

A graph similar to Fig. 2, with $\Delta_{\min }$ varied for different curves, is shown in Fig. 3. It is obvious that the disparity between the two temperature regimes becomes more pronounced as $\Delta_{\min }$ is lowered.

Figure 4 shows the temperature $T_{\text {co }}$ of the velocity maximum as a function of the asymmetry amplitude $\hat{\epsilon}$ and $\Delta_{\min }$, determined numerically from (3.30). In the linear response limit the maximum is located at a temperature near $T_{\omega}$ and is independent of $\Delta_{\min }$. For very high strain fields, a $\ln \hat{\epsilon}$ law for the strain dependence of $T_{\text {co }}$ is observed experimentally. This case, however, is outside the parameter range $\hbar \hat{\epsilon}<k T$ considered here.

We find a clear distinction between the acoustic properties of insulating glasses and those of amorphous metals (as described by the tunneling model). The charge fluctuations of conduction electrons destroy quantum coherence in the tunneling process on a time scale much shorter than probed by a vibrating-reed experiment, whereas quantum coherence is preserved in insulating glasses. These differences become especially relevant at low temperatures, where the regime of linear response is left for typical vibrating-reed experiments. For insulating

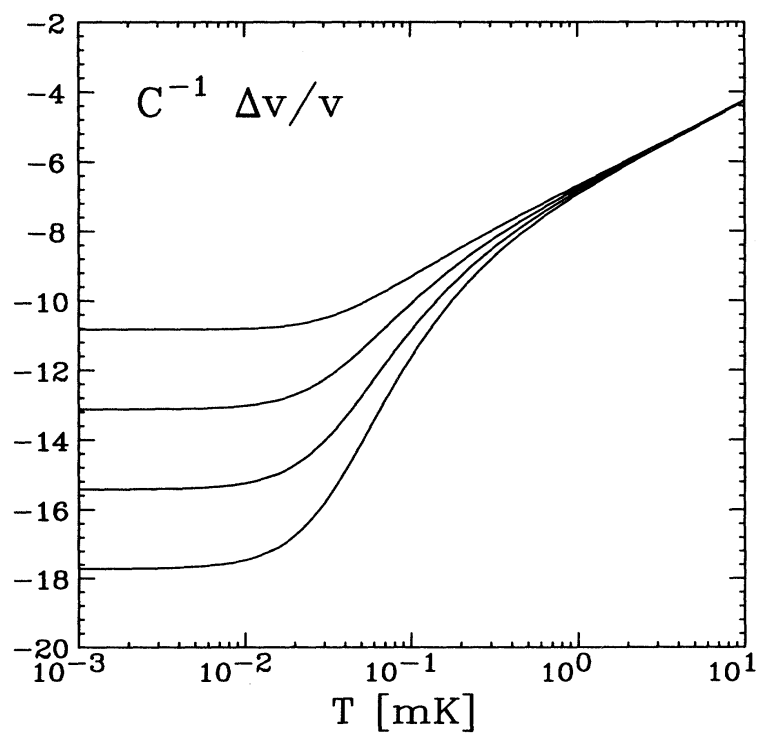

FIG. 3. Temperature dependence of the sound velocity of a insulating glass for different values of $\Delta_{\min }$. Curves are for $\hbar \Delta_{\min } / k=10^{-5}, 10^{-6}, 10^{-7}$, and $10^{-8} \mathrm{~K}$ (top to bottom), and for $\hbar \hat{\epsilon}=0.1 \mathrm{mK}$. 


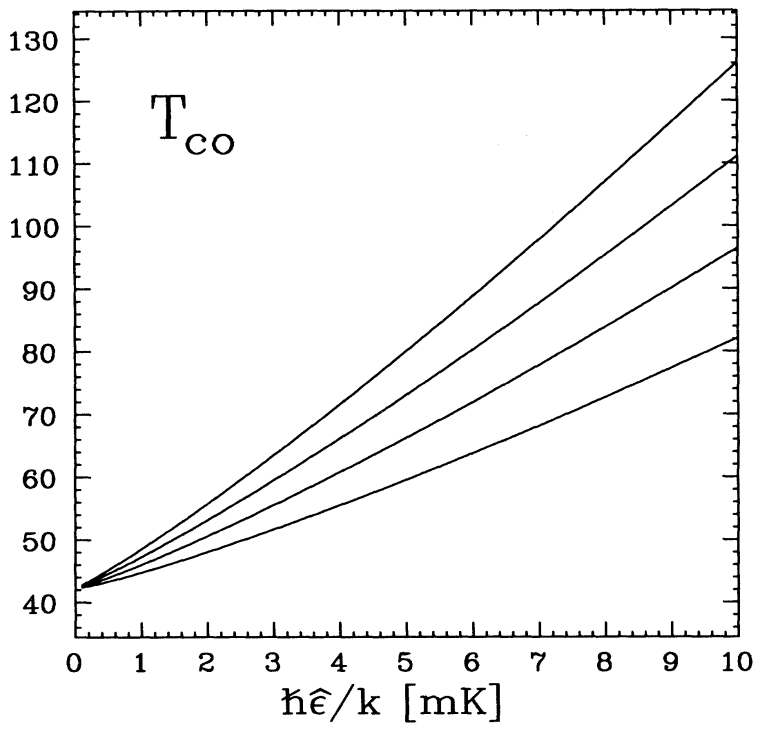

FIG. 4. Location of the velocity maximum as a function of strain level. The different values of $\Delta_{\min }$ are $10^{-5}, 10^{-6}$, $10^{-7}$, and $10^{-8} \mathrm{~K}$ (bottom to top), and $T_{\omega}=30 \mathrm{mK}$.

glasses, the acoustic response of a TS with arbitrarily small tunneling frequency remains finite, leading to a divergent response unless a lower bound for the distribution of tunneling frequencies is assumed. For TS in metals, on the other hand, the incoherent tunneling motion will be marginally slow in the limit of small $\Delta$, i.e., TS with a tunneling frequency below a threshold set by the reed frequency, amplitude, and temperature do not significantly affect the outcome of acoustic experiments. A smooth transition is therefore found between saturation region and linear response instead of the steep rise seen in insulating glasses.

On the whole, the coincidence between theory and experiment seems remarkable, given the complexity of the observed behavior and the crude estimates the TM is based on. We hope this will be considered another incentive to put the TM on firmer theoretical ground.

\section{ACKNOWLEDGMENTS}

We would like to thank G. Weiss and P. Esquinazi for interesting and stimulating discussions. This work was supported by the SCIENCE program of the European Union under Contract No. SC1*-CT91-0656 (TSTS).

\section{APPENDIX: NUMERICAL EVALUATION OF THE SOUND VELOCITY}

The cost of evaluating the integrations in (2.12), (2.7), and (3.8) can be significantly reduced by carefully considering the asymptotic behavior of the nonlinear susceptibility at very large or very small intrinsic energy scales. For convenience, all energies will be scaled by $\hat{\epsilon}$ in the following, using the definitions

$$
\begin{aligned}
\delta & =\frac{\Delta}{\hat{\epsilon}}, \\
b & =\frac{\epsilon_{0}}{\hat{\epsilon}}, \\
\vartheta & =\frac{k T}{\hbar \hat{\epsilon}} .
\end{aligned}
$$

With the nonlinear susceptibility given by $(2.7),(2.22)$, and (3.8), $\bar{\chi}$ assumes the form

$$
\bar{\chi}=\int_{\delta_{\min }}^{\delta_{\max }} \frac{d \delta}{\delta} \int_{0}^{b_{\max }} d b \frac{I_{1} I_{2}}{I_{3}}
$$

with

$$
\begin{aligned}
I_{1}= & \frac{1}{\pi} \int_{0}^{\pi} d x \cos x \frac{b+\cos x}{\sqrt{\delta^{2}+(b+\cos x)^{2}}}, \\
I_{2}= & \frac{1}{\pi} \int_{0}^{\pi} d x \sqrt{\delta^{2}+(b+\cos x)^{2}} \\
I_{3}= & \frac{1}{\pi} \int_{0}^{\pi} d x \sqrt{\delta^{2}+(b+\cos x)^{2}} \\
& \times \operatorname{coth} \frac{\sqrt{\delta^{2}+(b+\cos x)^{2}}}{2 \theta} .
\end{aligned}
$$

It is obvious that this complicated expression need not be evaluated in the asymptotic region of very large $\delta$ and $b$, where the linear response approximation is valid. A particularly simple integrand is obtained if the conditions $\delta \gg \vartheta, b \gg 1$, and $b \gg \vartheta$ are satisfied:

$$
\tilde{\chi}=\frac{1}{2 \hat{\epsilon}} \frac{\delta^{2}}{\left(\delta^{2}+b^{2}\right)^{3 / 2}} .
$$

For the case of small tunneling frequency $\delta \ll 1$, one may simplify the integrand of (A4) by using the expressions

$$
\begin{aligned}
& I_{1}^{\prime}=\frac{1}{\pi} \int_{0}^{\pi} d x \cos x \operatorname{sgn}(b+\cos x) \\
& I_{2}^{\prime}=\frac{1}{\pi} \int_{0}^{\pi} d x|b+\cos x|, \\
& I_{3}^{\prime}=\frac{1}{\pi} \int_{0}^{\pi} d x(b+\cos x) \operatorname{coth} \frac{b+\cos x}{2 \theta},
\end{aligned}
$$

instead of (A5) and (A6).

Splitting the integration range in an appropriate way allows one to take advantage of these simplifications. With good accuracy one can use (A8) for

$$
\begin{aligned}
& \delta>\delta_{1}=10 \vartheta \\
& b>b_{1}=10 \vartheta+10 .
\end{aligned}
$$

All of the plots shown in Figs. 2 and 3 were prepared using these values for $\delta_{1}$ and $b_{1}$. The low- $\delta$ limit (A9)(A11) was used for

$$
\delta<\delta^{*}=10^{-6}
$$

Thus one ends up with an expression for $\bar{\chi}$ that consists of three different terms,

$$
\bar{\chi}=A\left(\theta, \delta_{\max }\right)+I^{*}(\theta)+\ln \frac{\delta^{*}}{\delta_{\min }} J(\theta)
$$


where the function

$$
\begin{aligned}
A\left(\theta, \delta_{\max }\right) & =\frac{1}{2} \int_{\delta_{1}}^{\delta_{\max }} \frac{d \delta}{\delta} \int_{b_{1}}^{b_{\max }} d b \frac{\delta^{2}}{\left(\delta^{2}+b^{2}\right)^{3 / 2}} \\
& =\frac{1}{2} \ln \frac{2 \delta_{\max }}{b_{1}+\sqrt{\delta_{1}^{2}+b_{1}^{2}}}-\frac{1}{2}
\end{aligned}
$$

is known analytically, and the functions

$$
I^{*}(\theta)=\int_{\delta^{*}}^{\delta_{1}} \frac{d \delta}{\delta} \int_{0}^{\infty} d b \frac{I_{1} I_{2}}{I_{3}}+\int_{\delta_{1}}^{\infty} \frac{d \delta}{\delta} \int_{0}^{b_{1}} d b \frac{I_{1} I_{2}}{I_{3}}
$$

and

$$
\begin{aligned}
J(\theta) & =\int_{0}^{1} d b \frac{I_{1}^{\prime} I_{2}^{\prime}}{I_{3}^{\prime}} \\
& =\frac{2}{\pi} \int_{0}^{1} d b \frac{2\left(1-b^{2}\right)+[2 \arccos (-b)-\pi] b \sqrt{1-b^{2}}}{\int_{0}^{\pi} d x(b+\cos x) \operatorname{coth} \frac{b+\cos x}{2 \theta}}
\end{aligned}
$$

depend only on a single parameter. Once the functions $I^{*}(\theta)$ and $J(\theta)$ have been numerically obtained and tabulated, the sound velocity can be calculated from (A15) for varying parameters $T, \hat{\epsilon}, \Delta_{\min }$, and $\Delta_{\max }$ without having to reevaluate any numerical integration. Thus it should be easy to use our results in a least-squares fit of experimental data.
* Present address: Department of Chemistry, University of Southern California, Los Angeles, CA 90089-0482.

${ }^{1}$ P. W. Anderson, B. I. Halperin, and C. M. Varma, Philos. Mag. B 25, 1 (1972).

${ }^{2}$ W. A. Phillips, J. Low Temp. Phys. 7, 351 (1972).

${ }^{3}$ B. D. Laikhtman, Phys. Rev. B 29, 3601 (1984).

${ }^{4}$ Yu. M. Galperin, Sov. Phys. JETP 58, 804 (1983).

${ }^{5}$ P. Esquinazi, R. König, and F. Pobell, Z. Phys. B 87, 305 (1992).

${ }^{6} \mathrm{~J}$. Classen and G. Weiss, in Phonon Scattering in Condensed Matter VII, Springer Series in Solid State Sciences, Vol. 112, edited by M. Meissner and R. O. Pohl (Springer, Berlin, 1993).

${ }^{7}$ A. J. Leggett, S. Chakravarty, A. T. Dorsey, M. P. A. Fisher, A. Garg, and W. Zwerger, Rev. Mod. Phys. 59, 1 (1987), and references therein.

${ }^{8}$ D. A. Parshin, Z. Phys. B 91, 367 (1993).

${ }^{9}$ M. Grifoni, M. Sassetti, J. Stockburger, and U. Weiss, Phys. Rev. E 48, 3497 (1993).

${ }^{10}$ J. Stockburger, M. Grifoni, M. Sassetti, and U. Weiss, Z. Phys. B 94, 447 (1994).

${ }^{11}$ Given the excitation of a single extended mode only, the strain field $e(\mathbf{r}, t)$ and the stress $s(\mathbf{r}, t)$ can effectively be treated as scalar quantities.

12 J. Jäckle, Z. Phys. 257, 212 (1972).
${ }^{13}$ For a general discussion of dissipative quantum systems see, e.g., U. Weiss, Quantum Dissipative Systems, Series in Modern Condensed Matter Physics, Vol. 2 (World Scientific, Singapore, 1993).

${ }^{14}$ J. Kondo, in Fermi Surface Effects, Springer Series in Solid State Sciences, Vol. 77, edited by J. Kondo and A. Yoshimori (Springer, Berlin, 1988).

${ }^{15} \mathrm{~S}$. Hunklinger and A. K. Raychaudhury, in Progress in Low Temperature Physics, edited by D. F. Brewer (NorthHolland, Amsterdam, 1986), Vol. IX.

${ }^{16}$ B. Golding, J. E. Graebner, A. B. Kane, and J. L. Black, Phys. Rev. Lett. 41, 1487 (1978).

${ }^{17}$ J. L. Black, in Glassy Metals I, Topics in Applied Physics, Vol. 46, edited by H.-J. Güntherodt and H. Beck (Springer, Berlin, 1981), Chap. 8.

${ }^{18}$ Note that a fully quantitative comparison of our results to vibrating-reed data needs to take into account two minor complications. First, the strain field of a bending vibration is not homogeneous. One must therefore average the effect over the sample volume in order to predict the resonance shift of the bending vibration correctly. Second, the strain field varies due to the temperature dependence of the reed damping unless the driving force is adjusted accordingly. Both points have been addressed in detail in Ref. 10 . 